\title{
Upper and Lower Control Limits for Monitoring Markoff's Model and Non-Normal Variation with Known CV
}

\author{
Bhayare Unnati $^{1 *}$ and Singh Jais Raj ${ }^{2}$ \\ ${ }^{1 *}$ School of Studies in Statistics, Vikram University, Ujjain, India \\ ${ }^{2}$ School of Studies in Statistics, Vikram University, Ujjain, 456010, India \\ *Corresponding Author: unnati.b80@gmail.com
}

Available online at: www.isroset.org

Received:26/Oct/2017, Revised: 14/Nov/2017, Accepted: 12/Dec/2017, Published: 31/Dec/2017

\begin{abstract}
Control charts are essential tools for quality monitoring and construction of this charts were based on the assumption that observations were independent and normally distributed. The assumption of independence of quality characteristic is questionable, as autocorrelation among the observations becomes an inherent characteristic in quality control data. This phenomenon of inherent autocorrelation can cause significant deterioration of control charting performance. Autocorrelation had a substantial and detrimental effect on the statistical properties of control charts. The setting of control limits to utilize on a control chart assumes the assumption of normality. However, in many situation the condition does not hold. The objective of this paper is to investigate the upper and lower control limit properties of the $\overline{\mathrm{X}}$ control charts when the Markoff's model and non-normality exists in the process.
\end{abstract}

Keywords- Mean chart, Autocorrelation, Non-Normality, Markoff's Model, Coefficient of Variation

\section{INTRODUCTION}

Control charts were first proposed by Shewhart [32], and have become effective tools for improving process quality and productivity. A basic assumption in the applications of control charts is that the observations from the process at different times are independent random variables. However, the assumption of independence is often violated for processes in the chemical and pharmaceutical industries. Observations from these processes are always autocorrelated. When the control charts are developed under the independence assumption, the autocorrelated process results in decreasing the in-control average run length (ARL). For effective monitoring autocorrelated processes, one popular approach is to constructing control charts using the residuals from the time series model of the process data [2-13]. The properties of the proposed residual charts and their performance were investigated by Harris and Ross [12], Longnecker and Ryan [16], Yashchin [39], Kramer and Schmid [14], Schmid [27], Lin and Adams [15], Schmid [28], Padgett, Thombs and Padgett [23], Runger, Willemain and Prabhu [26], Vander Weil [34], Timmer, Pignatiello and Longnecker [33], Schmid [29], Zhang [40], Schmid and Schone [30], Alwan LC [2],Alwan and Roberts [3] and Lu and Reynolds [17]. Most of the papers on performance of control charts based on residuals has focused on the Shewhart control chart of residuals. The above papers on autocorrelated processes assume that imprecise measurement devices for process measurements are impossible. However, the performance of control charts and other statistical process control tools could be seriously affected when the process measurement includes the errors due to the measurement instrument. The effect of measurement error on the operating characteristics of and $\overline{\mathrm{X}}$ chart, in cases where only the process mean shifts, is discussed by Bennett [5], Mizuno [20], Abraham and Kartha [1]. Kanazuka [13] and Mittag [18] investigate the effect of measurement error on the power characteristics of the $\overline{\mathrm{X}}-\mathrm{R}$ control chart where both the process mean and process spreadchange. Mittag and Stemann [19] extend the results of Mittag[18], referring to the $\overline{\mathrm{X}}-\mathrm{S}$ control chart. Rahim [24] analyzed the effects of imprecise measurement devices on the design parameters of the economic $\bar{X}$ control chart, Bhayare and Singh [8] investigated the effect of measurement error on the power of $\bar{X}$ charts with known coefficient of variation.Yang [38] is worked the effect of measurement error on the design parameters of the economic asymmetric $\overline{\mathrm{X}}$ and $\mathrm{S}$ control charts. The above papers on control charts consider a single process control. Today, many industrial products are produced in several dependent processes. Consequently, it is not appropriate to monitor these processes by utilizing a control chart for each individual process. Zhang [41] proposes a simple cause selecting chart to monitor the second process of the two dependent processes. Wade and Woodall [35] reviewed the basic principles of the cause-selecting chart for two dependent processes and modify Zhang's approach, and give an example to illustrate the use of the individual X chart and the simple cause selecting chart. It is shown that their approach is better than that of Zhang for dependent processes control 
The objective of this paper is to investigate the upper and lower control limit properties of the $\overline{\mathrm{X}}$ control charts when the Markoff's model and non-normality exists in the process. The Markoff's model will be developed and studied. The nonnormal population is represented by considering first four terms of an Edgeworth series. An empirical example is given to calculate the tables for upper and lower control limits to illustrate the application and performance of the proposed control chart. The values of $\lambda_{3}$ and $\lambda_{4}$ considered are within Barton and Dennis [4] limits, which means that for such values the population is positive definite and uni-modal. Finally, a comparative study between Markoff's model and non-normality for $\overline{\mathrm{X}}$ chart will be conducted. Some conclusions will be drawn based on the comparison.

\section{The Markoff's Process for a Control Chart}

Suppose that observations are taken from Markoff's process. The $\mathrm{t}^{\text {th }}$ observation $\mathrm{x}_{\mathrm{t}}$ can be written as

$$
\mathrm{x}_{\mathrm{t}}=\mu+\xi_{\mathrm{t}}, \mathrm{t}=1,2,3, \ldots, \mathrm{n}
$$

where $\mu$ is the random process mean at sampling time $t$ and $\xi_{\mathrm{t}}$ is the random error. It is further assumed that $\xi_{\mathrm{t}}$ can be defined as the Markoff's model process and can be expressed by

$$
\xi_{\mathrm{t}}=\alpha_{1} \xi_{\mathrm{t}-1}+\varepsilon_{\mathrm{t}}
$$

where $\alpha_{1}$ is the autoregressive parameter which is the correlation measure between $\xi_{\mathrm{t}}$ and $\xi_{\mathrm{t}-1}$. The process is stationary if $\left|\alpha_{1}\right|$ $<1$. If $\alpha_{1}=0$, the random process means are independent. The variable $\epsilon_{\mathrm{t}}$ is a random shock which is normally distributed with mean 0 and variance $\sigma_{\varepsilon}^{2}$ and is independent of the other random errors and random shocks. The distribution of $\xi_{\mathrm{t}}$ for $\mathrm{t} \geq 1$ depends on starting value $\xi_{\mathrm{o}}$ for the series at starting time $\mathrm{t}_{\mathrm{o}}$. It is assumed that $\xi_{\mathrm{o}}$ is normally distributed with mean $\mu$ and variance $\sigma_{\xi}^{2}$. The value of $\xi_{t}$ follows a normal distribution with mean $\mu$ and variance $\sigma_{\xi}^{2}=\sigma_{\varepsilon}^{2} /\left(1-\alpha_{1}^{2}\right)$. Consequently, the distribution of $x_{t}$ has mean $\mu$ and variance $\sigma_{x}^{2}=\sigma_{\xi}^{2}+\sigma_{\varepsilon}^{2}$. The value of $\sigma_{\xi}^{2}$ can be considered as a long-term variability and $\sigma_{\varepsilon}^{2}$ can be represented as a combination of short-term variability and measurement error. When $\alpha_{1}=0$ (i. e., $\left.\sigma_{\xi}^{2}=0\right)$, model (2) is the independent case (i. e., $X_{t}=\mu+\varepsilon_{t}$, where $\mu$ is constant). For Markoff's process the mean and variance of $\overline{\mathbf{X}}$ is given by

$$
\begin{aligned}
& \mathrm{E}(\overline{\mathrm{X}})=\mu \\
& \operatorname{Var}(\overline{\mathrm{x}})=\frac{\sigma^{2}}{\mathrm{n}}\left[\frac{1+\alpha_{1}}{1-\alpha_{1}}-\frac{2 \alpha_{1}}{\mathrm{n}} \frac{\left(1-\alpha_{1}^{\mathrm{n}}\right)}{\left(1-\alpha_{1}\right)^{2}}\right]=\frac{\sigma^{2}}{\mathrm{n}} \lambda\left(\alpha_{1}, \mathrm{n}\right) \text { Say }
\end{aligned}
$$

\section{THE EFFECTS OF MARKOFF'S MODEL AND NON-NORMALITY ON CONTROL LIMITS FOR MEANS}

The control limits for mean chart with known cv calculated on the basis of normal population may be seriously affected particularly in cases of variations showing significant departures of $\lambda_{3}$ and $\lambda_{4}$ from their respective normal theory values and Markoff's process. For a random sample of $n$ observation $x_{1}, x_{2}, \ldots, x_{n}$ an estimator $\bar{x}^{\prime}=w \sum_{t=1}^{n} x_{t}$ has been constructed by Searls[31]. Where

$$
\omega=\frac{1}{\left[n+\lambda\left(\alpha_{1}, n\right) v^{2}\right]} \text { and }
$$




$$
\operatorname{Min} . \operatorname{MSE}\left(\bar{x}^{\prime}\right)=\frac{\sigma^{2}}{\left[\frac{n}{\lambda\left(\alpha_{1}, n\right)}+v^{2}\right]} .
$$

Let $\eta$ denote the variable for the quality characteristic in standard form i.e., $\eta=(x-\mu) / \sigma$. The p percent probability level of the standard normal variable $\eta$ by the first four terms of Edgeworth series and Markoff's model can be expressed as

$$
\begin{gathered}
\eta=x+\frac{1}{6} \lambda_{3} T\left(x^{2}-1\right)+\frac{1}{24} \lambda_{4} T^{2}\left(x^{3}-3 x\right)-\frac{1}{36} \lambda_{3}^{2} T^{2}\left(2 x^{3}-5 x\right) \\
=x+\lambda_{3} T_{3}(x)+\lambda_{4} T^{2} M_{4}(x)+\lambda_{3}^{2} T^{2} M_{33}(x)
\end{gathered}
$$

Where $M_{3}(x)=\frac{1}{6}\left(x^{2}-1\right), M_{4}(x)=\frac{1}{24}\left(x^{3}-3 x\right), M_{33}(x)=-\frac{1}{36}\left(2 x^{3}-5 x\right)$ and $T^{2}=\left(\frac{1}{\lambda\left(\alpha_{1}, n\right)}+\frac{v^{2}}{n}\right)$. We note that the probability integrals for non-normal variable $\eta$ as

$$
\int_{-\infty}^{\eta_{1}} p(x) d x=\int_{\eta_{2}}^{\infty} p(x) d x=0.005 \text {. }
$$

In which $\eta_{1}$ and $\eta_{2}$ are given by

$\bar{\eta}=\bar{x}+\lambda_{3} \mathrm{TM}_{3}\left(\overline{\mathrm{x}}^{\prime}\right)+\lambda_{4} \mathrm{~T}^{2} \mathrm{M}_{4}\left(\overline{\mathrm{x}}^{\prime}\right)+\lambda_{3} \mathrm{~T}^{2} \mathrm{M}_{33}\left(\overline{\mathrm{x}}^{\prime}\right)$,

Where $\overline{\mathrm{x}}^{\prime}= \pm 2.576 / \mathrm{T}$.

\section{NUMERICAL ILLUSTRATION AND CONCLUSION}

For various non-normal populations with various non-normality parameters $\left(\lambda_{3}, \lambda_{4}\right)$ and for different values of autocorrelation with varying known cv the values of upper and lower control limits are given in Table 1 to 4 . For many continuous processes, there may correlation between observations that are closely spaced in time. It is observed that a control chart using various sample sizes always leads to different level of autocorrelation between consecutive samples. As the level of autocorrelation increases the difference between the limits are larger for positive autocorrelation, which indicates that it takes more time and more sample to detect the mean shifts. Based on the study, for the case of $\alpha_{1}$ close to 0 , i.e., close to the independent case, the limits of $\overline{\mathbf{X}}$-chart is generally better than Markoff's model in detecting small mean shift. When autocorrelation is negative, the difference between the limits are reduced rapidly, which is good feature for apply $\overline{\mathrm{X}}$-chart. By comparing entries of Table 1 for independent case $\left(\alpha_{1}=0\right)$ with Table 2 to Table 4 when $\mathrm{v}=0,2,4,6$, one can easily see that the effect of autocorrelation is quite serious on the lower and upper control limits.

Table-1: Upper and lower Control Limits for Non-Normal Population with Known cv Under Markoff's model $\left(\alpha_{1}=0.0\right)$

\begin{tabular}{|c|c|c|c|c|c|r|r|r|r|r|}
\hline \multicolumn{9}{|c|}{$(\mathbf{v}=\mathbf{0})$} & \multicolumn{5}{|c|}{$(\mathbf{v}=\mathbf{2})$} \\
\hline $\boldsymbol{\lambda}_{\mathbf{3}} \rightarrow$ & $\mathbf{- 1}$ & $\mathbf{- 0 . 5}$ & 0 & $\mathbf{0 . 5}$ & $\mathbf{1}$ & $\mathbf{- 1}$ & $\mathbf{- 0 . 5}$ & $\mathbf{0}$ & $\mathbf{0 . 5}$ & $\mathbf{1}$ \\
$\boldsymbol{\lambda}_{\mathbf{4} \downarrow}$ & & & & & & & & & & \\
$\mathbf{- 0 . 5}$ & -2.8863 & -2.8861 & -2.5405 & -1.8499 & -0.8136 & -2.4063 & -2.3178 & -2.0469 & -1.6086 & -1.0179 \\
& 0.8133 & 1.8496 & 2.5405 & 2.8861 & 2.8863 & 0.9578 & 1.5936 & 2.0469 & 2.3178 & 2.4063 \\
\hline \multirow{2}{*}{$\mathbf{0 . 3}$} & -2.9799 & -2.9797 & -2.6342 & -1.9435 & -0.9073 & -2.4482 & -2.3597 & -2.0888 & -1.6505 & -1.0598 \\
& 0.9070 & 1.9432 & 2.6342 & 2.9797 & 2.9799 & 0.9997 & 1.6355 & 2.0888 & 2.3597 & 2.4482 \\
\hline \multirow{2}{*}{$\mathbf{0 . 1}$} & -3.0735 & -3.0733 & -2.7278 & -2.0372 & -1.0009 & -2.4901 & -2.4016 & -2.1307 & -1.6924 & -1.1017 \\
& 1.0006 & 2.0369 & 2.7278 & 3.0733 & 3.0735 & 1.0416 & 1.6773 & 2.1307 & 2.4016 & 2.4901 \\
\hline
\end{tabular}




\begin{tabular}{|c|c|c|c|c|c|c|c|c|c|c|}
\hline \multirow{2}{*}{$\mathbf{0}$} & -3.1203 & -3.1202 & -2.7746 & -2.0840 & -1.0477 & -2.5110 & -2.4225 & -2.1516 & -1.7133 & -1.1226 \\
\hline & 1.0474 & 2.0837 & 2.7746 & 3.1202 & 3.1203 & 1.0626 & 1.6983 & 2.1516 & 2.4225 & 2.5110 \\
\hline \multirow{2}{*}{0.1} & -3.1672 & -3.1670 & -2.8214 & -2.1308 & -1.0945 & -2.5320 & -2.4435 & -2.1725 & -1.7342 & -1.1436 \\
\hline & 1.0942 & 2.1305 & 2.8214 & 3.1670 & 3.1672 & 1.0835 & 1.7192 & 2.1725 & 2.4435 & 2.5320 \\
\hline \multirow{2}{*}{0.3} & -3.2608 & -3.2606 & -2.9150 & -2.2244 & -1.1882 & -2.5739 & -2.4853 & -2.2144 & -1.7761 & -1.1855 \\
\hline & 1.1879 & 2.2241 & 2.9150 & 3.2606 & 3.2608 & 1.1254 & 1.7611 & 2.2144 & 2.4853 & 2.5739 \\
\hline \multirow{2}{*}{0.5} & -3.3544 & -3.3542 & -3.0087 & -2.3181 & -1.2818 & -2.6158 & -2.5272 & -2.2563 & -1.8180 & -1.2274 \\
\hline & 1.2815 & 2.3178 & 3.0087 & 3.3542 & 3.3544 & 1.1673 & 1.8030 & 2.2563 & 2.5272 & 2.6158 \\
\hline \multirow{2}{*}{0.7} & -3.4481 & -3.4479 & -3.1023 & -2.4117 & -1.3754 & -2.6576 & -2.5691 & -2.2982 & -1.8599 & -1.2692 \\
\hline & 1.3751 & 2.4114 & 3.1023 & 3.4479 & 3.4481 & 1.2091 & 1.8449 & 2.2982 & 2.5691 & 2.6576 \\
\hline \multirow{2}{*}{0.9} & -3.5417 & -3.5415 & -3.1959 & -2.5053 & -1.4691 & -2.6995 & -2.6110 & -2.3401 & -1.9018 & -1.3111 \\
\hline & 1.4688 & 2.5050 & 3.1959 & 3.5415 & 3.5417 & 1.2510 & 1.8868 & 2.3401 & 2.6110 & 2.6995 \\
\hline
\end{tabular}

\begin{tabular}{|c|r|r|r|r|r|r|r|r|r|r|}
\hline \multicolumn{9}{|c|}{$(\mathbf{v}=\mathbf{4})$} & \multicolumn{5}{|c|}{ (v=6) } \\
\hline $\boldsymbol{\lambda}_{\mathbf{3}} \rightarrow$ & $\mathbf{- 1}$ & $\mathbf{- 0 . 5}$ & 0 & $\mathbf{0 . 5}$ & $\mathbf{1}$ & $\mathbf{- 1}$ & $\mathbf{- 0 . 5}$ & $\mathbf{0}$ & $\mathbf{0 . 5}$ & $\mathbf{1}$ \\
$\boldsymbol{\lambda}_{\mathbf{4} \downarrow}$ & & & & & & & & & \\
\hline $\mathbf{0 . 5}$ & -1.9640 & -1.7256 & -1.5369 & -1.3979 & -1.3087 & -1.8327 & -1.4442 & -1.3004 & -1.4013 & -1.7469 \\
& 1.3087 & 1.3979 & 1.5369 & 1.7256 & 1.9640 & 1.7469 & 1.4013 & 1.3004 & 1.4442 & 1.8327 \\
\hline \multirow{2}{*}{$\mathbf{0 . 3}$} & -1.9301 & -1.6916 & -1.5029 & -1.3639 & -1.2747 & -1.7330 & -1.3445 & -1.2007 & -1.3016 & -1.6472 \\
& 1.2747 & 1.3639 & 1.5029 & 1.6916 & 1.9301 & 1.6472 & 1.3016 & 1.2007 & 1.3445 & 1.7330 \\
\hline \multirow{2}{*}{$\mathbf{0 . 1}$} & -1.8961 & -1.6576 & -1.4689 & -1.3299 & -1.2407 & -1.6333 & -1.2448 & -1.1010 & -1.2019 & -1.5476 \\
& 1.2407 & 1.3299 & 1.4689 & 1.6576 & 1.8961 & 1.5476 & 1.2019 & 1.1010 & 1.2448 & 1.6333 \\
\hline \multirow{2}{*}{$\mathbf{0}$} & -1.8791 & -1.6406 & -1.4519 & -1.3129 & -1.2237 & -1.5835 & -1.1950 & -1.0512 & -1.1521 & -1.4977 \\
& 1.2237 & 1.3129 & 1.4519 & 1.6406 & 1.8791 & 1.4977 & 1.1521 & 1.0512 & 1.1950 & 1.5835 \\
\hline \multirow{2}{*}{$\mathbf{0 . 1}$} & -1.8621 & -1.6236 & -1.4349 & -1.2959 & -1.2067 & -1.5336 & -1.1452 & -1.0014 & -1.1023 & -1.4479 \\
& 1.2067 & 1.2959 & 1.4349 & 1.6236 & 1.8621 & 1.4479 & 1.1023 & 1.0014 & 1.1452 & 1.5336 \\
\hline \multirow{2}{*}{$\mathbf{0 . 3}$} & -1.8281 & -1.5896 & -1.4009 & -1.2619 & -1.1727 & -1.4340 & -1.0455 & -0.9017 & -1.0026 & -1.3482 \\
& 1.1727 & 1.2619 & 1.4009 & 1.5896 & 1.8281 & 1.3482 & 1.0026 & 0.9017 & 1.0455 & 1.4340 \\
\hline \multirow{2}{*}{$\mathbf{0 . 5}$} & -1.7941 & -1.5556 & -1.3669 & -1.2279 & -1.1387 & -1.3343 & -0.9458 & -0.8020 & -0.9029 & -1.2485 \\
& 1.1387 & 1.2279 & 1.3669 & 1.5556 & 1.7941 & 1.2485 & 0.9029 & 0.8020 & 0.9458 & 1.3343 \\
\hline \multirow{2}{*}{$\mathbf{0 . 7}$} & -1.7601 & -1.5216 & -1.3329 & -1.1939 & -1.1047 & -1.2346 & -0.8461 & -0.7023 & -0.8032 & -1.1488 \\
& 1.1047 & 1.1939 & 1.3329 & 1.5216 & 1.7601 & 1.1488 & 0.8032 & 0.7023 & 0.8461 & 1.2346 \\
\hline \multirow{2}{*}{$\mathbf{0 . 9}$} & -1.7261 & -1.4876 & -1.2989 & -1.1599 & -1.0707 & -1.1349 & -0.7464 & -0.6026 & -0.7035 & -1.0491 \\
& 1.0707 & 1.1599 & 1.2989 & 1.4876 & 1.7261 & 1.0491 & 0.7035 & 0.6026 & 0.7464 & 1.1349 \\
\hline
\end{tabular}

Table-2: Upper and lower Control Limits for Non-Normal Population with Known cv Under Markoff's model $\left(\alpha_{1}=-0.5\right)$

\begin{tabular}{|c|l|l|l|l|l|l|l|l|l|c|c|}
\hline \multicolumn{9}{|c|}{$(\mathbf{v}=\mathbf{0})$} & \multicolumn{5}{|c|}{$(\mathbf{v}=\mathbf{2})$} \\
\hline $\boldsymbol{\lambda}_{\mathbf{3}} \rightarrow$ & $\mathbf{- 1}$ & $\mathbf{- 0 . 5}$ & $\mathbf{0}$ & $\mathbf{0 . 5}$ & $\mathbf{1}$ & $\mathbf{- 1}$ & $\mathbf{- 0 . 5}$ & $\mathbf{0}$ & $\mathbf{0 . 5}$ & $\mathbf{1}$ \\
\hline $\boldsymbol{\lambda}_{\mathbf{4} \downarrow}$ & & & & & & & & & & \\
\hline $\mathbf{- 0 . 5}$ & 1.1910 & 1.4306 & 1.6547 & 1.8633 & 2.0564 & 1.2980 & 1.4000 & 1.5461 & 1.7364 & 1.9709 \\
& -2.0564 & -1.8633 & -1.6547 & -1.4306 & -1.1910 & -1.9709 & -1.7364 & -1.5461 & -1.4000 & -1.2980 \\
\hline \multirow{2}{*}{$\mathbf{0 . 3}$} & 1.1786 & 1.4182 & 1.6423 & 1.8509 & 2.0440 & 1.2659 & 1.3679 & 1.5140 & 1.7043 & 1.9388 \\
& -2.0440 & -1.8509 & -1.6423 & -1.4182 & -1.1786 & -1.9388 & -1.7043 & -1.5140 & -1.3679 & -1.2659 \\
\hline \multirow{2}{*}{$\mathbf{0 . 1}$} & 1.1662 & 1.4058 & 1.6299 & 1.8385 & 2.0316 & 1.2338 & 1.3358 & 1.4819 & 1.6722 & 1.9067 \\
& -2.0316 & -1.8385 & -1.6299 & -1.4058 & -1.1662 & -1.9067 & -1.6722 & -1.4819 & -1.3358 & -1.2338 \\
\hline \multirow{2}{*}{$\mathbf{0}$} & 1.1600 & 1.3996 & 1.6237 & 1.8323 & 2.0255 & 1.2178 & 1.3197 & 1.4658 & 1.6562 & 1.8906 \\
& -2.0255 & -1.8323 & -1.6237 & -1.3996 & -1.1600 & -1.8906 & -1.6562 & -1.4658 & -1.3197 & -1.2178 \\
\hline \multirow{2}{*}{$\mathbf{0 . 1}$} & 1.1538 & 1.3934 & 1.6176 & 1.8262 & 2.0193 & 1.2017 & 1.3037 & 1.4498 & 1.6401 & 1.8746 \\
& -2.0193 & -1.8262 & -1.6176 & -1.3934 & -1.1538 & -1.8746 & -1.6401 & -1.4498 & -1.3037 & -1.2017 \\
\hline \multirow{2}{*}{$\mathbf{0 . 3}$} & 1.1415 & 1.3811 & 1.6052 & 1.8138 & 2.0069 & 1.1696 & 1.2715 & 1.4177 & 1.6080 & 1.8425 \\
& -2.0069 & -1.8138 & -1.6052 & -1.3811 & -1.1415 & -1.8425 & -1.6080 & -1.4177 & -1.2715 & -1.1696 \\
\hline
\end{tabular}


Int. J. Sci. Res. in Mathematical and Statistical Sciences

Vol. 4(6), Dec 2017, ISSN: 2348-4519

\begin{tabular}{|c|l|l|l|l|l|l|l|l|c|c|}
$\mathbf{0 . 5}$ & 1.1291 & 1.3687 & 1.5928 & 1.8014 & 1.9945 & 1.1375 & 1.2394 & 1.3856 & 1.5759 & 1.8103 \\
& -1.9945 & -1.8014 & -1.5928 & -1.3687 & -1.1291 & -1.8103 & -1.5759 & -1.3856 & -1.2394 & -1.1375 \\
\hline \multirow{2}{*}{$\mathbf{0 . 7}$} & 1.1167 & 1.3563 & 1.5804 & 1.7890 & 1.9821 & 1.1053 & 1.2073 & 1.3534 & 1.5437 & 1.7782 \\
& -1.9821 & -1.7890 & -1.5804 & -1.3563 & -1.1167 & -1.7782 & -1.5437 & -1.3534 & -1.2073 & -1.1053 \\
\hline \multirow{2}{*}{$\mathbf{0 . 9}$} & 1.1043 & 1.3439 & 1.5680 & 1.7766 & 1.9698 & 1.0732 & 1.1752 & 1.3213 & 1.5116 & 1.7461 \\
& -1.9698 & -1.7766 & -1.5680 & -1.3439 & -1.1043 & -1.7461 & -1.5116 & -1.3213 & -1.1752 & -1.0732 \\
\hline
\end{tabular}

\begin{tabular}{|c|c|c|c|l|l|l|l|l|l|l|}
\hline \multicolumn{9}{|c|}{$(\mathbf{v}=\mathbf{4})$} & \multicolumn{1}{c|}{$\mathbf{( v = 6 )}$} \\
\hline$\lambda_{\mathbf{3}} \rightarrow$ & $\mathbf{- 1}$ & $\mathbf{- 0 . 5}$ & $\mathbf{0}$ & $\mathbf{0 . 5}$ & $\mathbf{1}$ & $\mathbf{- 1}$ & $\mathbf{- 0 . 5}$ & $\mathbf{0}$ & $\mathbf{0 . 5}$ & $\mathbf{1}$ \\
\hline $\boldsymbol{\lambda} \downarrow$ & & & & & & & & \\
\hline \multirow{2}{*}{$\mathbf{0 . 5}$} & 1.5771 & 1.3838 & 1.3658 & 1.5232 & 1.8559 & 1.9564 & 1.4403 & 1.2477 & 1.3786 & 1.8331 \\
& -1.8559 & -1.5232 & -1.3658 & -1.3838 & -1.5771 & -1.8331 & -1.3786 & -1.2477 & -1.4403 & -1.9564 \\
\hline \multirow{2}{*}{$\mathbf{0 . 3}$} & 1.5010 & 1.3076 & 1.2897 & 1.4470 & 1.7798 & 1.8296 & 1.3135 & 1.1209 & 1.2518 & 1.7063 \\
& -1.7798 & -1.4470 & -1.2897 & -1.3076 & -1.5010 & -1.7063 & -1.2518 & -1.1209 & -1.3135 & -1.8296 \\
\hline \multirow{2}{*}{$\mathbf{0 . 1}$} & 1.4248 & 1.2315 & 1.2135 & 1.3709 & 1.7037 & 1.7029 & 1.1867 & 0.9941 & 1.1251 & 1.5795 \\
& -1.7037 & -1.3709 & -1.2135 & -1.2315 & -1.4248 & -1.5795 & -1.1251 & -0.9941 & -1.1867 & -1.7029 \\
\hline \multirow{2}{*}{$\mathbf{0}$} & 1.3868 & 1.1934 & 1.1755 & 1.3329 & 1.6656 & 1.6395 & 1.1234 & 0.9308 & 1.0617 & 1.5162 \\
& -1.6656 & -1.3329 & -1.1755 & -1.1934 & -1.3868 & -1.5162 & -1.0617 & -0.9308 & -1.1234 & -1.6395 \\
\hline \multirow{2}{*}{$\mathbf{0 . 1}$} & 1.3487 & 1.1554 & 1.1374 & 1.2948 & 1.6275 & 1.5761 & 1.0600 & 0.8674 & 0.9983 & 1.4528 \\
& -1.6275 & -1.2948 & -1.1374 & -1.1554 & -1.3487 & -1.4528 & -0.9983 & -0.8674 & -1.0600 & -1.5761 \\
\hline \multirow{2}{*}{$\mathbf{0 . 3}$} & 1.2726 & 1.0792 & 1.0613 & 1.2187 & 1.5514 & 1.4493 & 0.9332 & 0.7406 & 0.8716 & 1.3260 \\
& -1.5514 & -1.2187 & -1.0613 & -1.0792 & -1.2726 & -1.3260 & -0.8716 & -0.7406 & -0.9332 & -1.4493 \\
\hline \multirow{2}{*}{$\mathbf{0 . 5}$} & 1.1964 & 1.0031 & 0.9851 & 1.1425 & 1.4753 & 1.3226 & 0.8064 & 0.6139 & 0.7448 & 1.1993 \\
& -1.4753 & -1.1425 & -0.9851 & -1.0031 & -1.1964 & -1.1993 & -0.7448 & -0.6139 & -0.8064 & -1.3226 \\
\hline \multirow{2}{*}{$\mathbf{0 . 7}$} & 1.1203 & 0.9270 & 0.9090 & 1.0664 & 1.3991 & 1.1958 & 0.6797 & 0.4871 & 0.6180 & 1.0725 \\
& -1.3991 & -1.0664 & -0.9090 & -0.9270 & -1.1203 & -1.0725 & -0.6180 & -0.4871 & -0.6797 & -1.1958 \\
\hline \multirow{2}{*}{$\mathbf{0 . 9}$} & 1.0442 & 0.8508 & 0.8329 & 0.9903 & 1.3230 & 1.0690 & 0.5529 & 0.3603 & 0.4913 & 0.9457 \\
& -1.3230 & -0.9903 & -0.8329 & -0.8508 & -1.0442 & -0.9457 & -0.4913 & -0.3603 & -0.5529 & -1.0690 \\
\hline
\end{tabular}

Table-3: Upper and lower Control Limits for Non-Normal Population with Known cv Under Markoff's model $\left(\alpha_{1}=0.5\right)$

\begin{tabular}{|c|c|c|c|c|c|c|c|c|c|c|}
\hline \multicolumn{9}{|c|}{$(\mathbf{v}=\mathbf{0})$} & \multicolumn{6}{c|}{$\mathbf{( v = 2 )}$} \\
\hline$\lambda_{\mathbf{3}} \rightarrow$ & $\mathbf{- 1}$ & $\mathbf{- 0 . 5}$ & $\mathbf{0}$ & $\mathbf{0 . 5}$ & $\mathbf{1}$ & $\mathbf{- 1}$ & $\mathbf{- 0 . 5}$ & $\mathbf{0}$ & $\mathbf{0 . 5}$ & $\mathbf{1}$ \\
\hline $\mathbf{2} \downarrow$ & & & & & & & & \\
\hline \multirow{2}{*.5}{} & 0.6957 & 2.4437 & 3.5658 & 4.0620 & 3.9322 & 0.8451 & 1.7730 & 2.3992 & 2.7236 & 2.7463 \\
& -3.9322 & -4.0620 & -3.5658 & -2.4437 & -0.6957 & -2.7463 & -2.7236 & -2.3992 & -1.7730 & -0.8451 \\
\hline \multirow{2}{*}{$\mathbf{0 . 3}$} & 0.8766 & 2.6246 & 3.7467 & 4.2429 & 4.1131 & 0.9250 & 1.8529 & 2.4791 & 2.8035 & 2.8262 \\
& -4.1131 & -4.2429 & -3.7467 & -2.6246 & -0.8766 & -2.8262 & -2.8035 & -2.4791 & -1.8529 & -0.9250 \\
\hline \multirow{2}{*}{$\mathbf{0 . 1}$} & 1.0575 & 2.8055 & 3.9276 & 4.4238 & 4.2940 & 1.0049 & 1.9328 & 2.5590 & 2.8834 & 2.9061 \\
& -4.2940 & -4.4238 & -3.9276 & -2.8055 & -1.0575 & -2.9061 & -2.8834 & -2.5590 & -1.9328 & -1.0049 \\
\hline \multirow{2}{*}{$\mathbf{0}$} & 1.1479 & 2.8960 & 4.0181 & 4.5142 & 4.3844 & 1.0448 & 1.9727 & 2.5989 & 2.9234 & 2.9460 \\
& -4.3844 & -4.5142 & -4.0181 & -2.8960 & -1.1479 & -2.9460 & -2.9234 & -2.5989 & -1.9727 & -1.0448 \\
\hline \multirow{2}{*}{$\mathbf{0 . 1}$} & 1.2384 & 2.9864 & 4.1086 & 4.6047 & 4.4749 & 1.0848 & 2.0127 & 2.6389 & 2.9633 & 2.9860 \\
& -4.4749 & -4.6047 & -4.1086 & -2.9864 & -1.2384 & -2.9860 & -2.9633 & -2.6389 & -2.0127 & -1.0848 \\
\hline \multirow{2}{*}{$\mathbf{0 . 3}$} & 1.4193 & 3.1674 & 4.2895 & 4.7856 & 4.6558 & 1.1647 & 2.0926 & 2.7187 & 3.0432 & 3.0659 \\
& -4.6558 & -4.7856 & -4.2895 & -3.1674 & -1.4193 & -3.0659 & -3.0432 & -2.7187 & -2.0926 & -1.1647 \\
\hline \multirow{2}{*}{$\mathbf{0 . 5}$} & 1.6002 & 3.3483 & 4.4704 & 4.9665 & 4.8367 & 1.2445 & 2.1725 & 2.7986 & 3.1231 & 3.1458 \\
& -4.8367 & -4.9665 & -4.4704 & -3.3483 & -1.6002 & -3.1458 & -3.1231 & -2.7986 & -2.1725 & -1.2445 \\
\hline \multirow{2}{*}{$\mathbf{0 . 7}$} & 1.7811 & 3.5292 & 4.6513 & 5.1474 & 5.0176 & 1.3244 & 2.2523 & 2.8785 & 3.2029 & 3.2256 \\
& -5.0176 & -5.1474 & -4.6513 & -3.5292 & -1.7811 & -3.2256 & -3.2029 & -2.8785 & -2.2523 & -1.3244 \\
\hline \multirow{2}{*}{$\mathbf{0 . 9}$} & 1.9620 & 3.7101 & 4.8322 & 5.3283 & 5.1985 & 1.4043 & 2.3322 & 2.9584 & 3.2828 & 3.3055 \\
& -5.1985 & -5.3283 & -4.8322 & -3.7101 & -1.9620 & -3.3055 & -3.2828 & -2.9584 & -2.3322 & -1.4043 \\
\hline
\end{tabular}




\begin{tabular}{|c|c|c|c|c|c|c|c|c|c|c|}
\hline \multicolumn{6}{|c|}{$(v=4)$} & \multicolumn{5}{|c|}{$(v=6)$} \\
\hline $\begin{array}{r}\lambda_{3} \rightarrow \\
\lambda_{4} \downarrow\end{array}$ & -1 & -0.5 & 0 & 0.5 & 1 & -1 & -0.5 & 0 & 0.5 & 1 \\
\hline-0.5 & $\begin{array}{l}1.2256 \\
-2.0252\end{array}$ & $\begin{array}{l}1.4186 \\
-1.8184\end{array}$ & $\begin{array}{l}1.6162 \\
-1.6162\end{array}$ & $\begin{array}{c}1.8184 \\
-1.4186\end{array}$ & $\begin{array}{c}2.0252 \\
-1.2256\end{array}$ & $\begin{array}{r}1.6851 \\
-1.8381\end{array}$ & $\begin{array}{r}1.3932 \\
-1.4697\end{array}$ & $\begin{array}{l}1.3214 \\
-1.3214\end{array}$ & $\begin{array}{c}1.4697 \\
-1.3932\end{array}$ & $\begin{array}{r}1.8381 \\
-1.6851\end{array}$ \\
\hline-0.3 & $\begin{array}{l}1.2066 \\
-2.0062\end{array}$ & $\begin{array}{l}1.3996 \\
-1.7994\end{array}$ & $\begin{array}{l}1.5972 \\
-1.5972\end{array}$ & $\begin{array}{c}1.7994 \\
-1.3996\end{array}$ & $\begin{array}{c}2.0062 \\
-1.2066\end{array}$ & $\begin{array}{r}1.5937 \\
-1.7468\end{array}$ & $\begin{array}{r}1.3018 \\
-1.3784\end{array}$ & $\begin{array}{l}1.2301 \\
-1.2301\end{array}$ & $\begin{array}{c}1.3784 \\
-1.3018\end{array}$ & $\begin{array}{c}1.7468 \\
-1.5937\end{array}$ \\
\hline-0.1 & $\begin{array}{l}1.1876 \\
-1.9872\end{array}$ & $\begin{array}{l}1.3806 \\
-1.7804\end{array}$ & $\begin{array}{l}1.5782 \\
-1.5782\end{array}$ & $\begin{array}{c}1.7804 \\
-1.3806\end{array}$ & $\begin{array}{c}1.9872 \\
-1.1876\end{array}$ & $\begin{array}{l}1.5024 \\
-1.6554\end{array}$ & $\begin{array}{l}1.2105 \\
-1.2870\end{array}$ & $\begin{array}{l}1.1387 \\
-1.1387\end{array}$ & $\begin{array}{r}1.2870 \\
-1.2105\end{array}$ & $\begin{array}{c}1.6554 \\
-1.5024\end{array}$ \\
\hline 0 & $\begin{array}{l}1.1781 \\
-1.9777\end{array}$ & $\begin{array}{l}1.3711 \\
-1.7709\end{array}$ & $\begin{array}{l}1.5687 \\
-1.5687\end{array}$ & $\begin{array}{r}1.7709 \\
-1.3711\end{array}$ & $\begin{array}{r}1.9777 \\
-1.1781\end{array}$ & $\begin{array}{l}1.4568 \\
-1.6098\end{array}$ & $\begin{array}{l}1.1649 \\
-1.2414\end{array}$ & $\begin{array}{l}1.0931 \\
-1.0931\end{array}$ & $\begin{array}{c}1.2414 \\
-1.1649\end{array}$ & $\begin{array}{c}1.6098 \\
-1.4568\end{array}$ \\
\hline 0.1 & $\begin{array}{l}1.1686 \\
-1.9682\end{array}$ & & $\begin{array}{l}1.5591 \\
-1.5591\end{array}$ & & $\begin{array}{c}1.9682 \\
-1.1686\end{array}$ & $\begin{array}{l}1.4111 \\
-1.5641\end{array}$ & $\begin{array}{l}1.1192 \\
-1.1957\end{array}$ & $\begin{array}{l}1.0474 \\
-1.0474\end{array}$ & $\begin{array}{c}1.1957 \\
-1.1192\end{array}$ & $\begin{array}{c}1.5641 \\
-1.4111\end{array}$ \\
\hline 0.3 & $\begin{array}{l}1.1496 \\
-1.9492\end{array}$ & $\begin{array}{l}1.3426 \\
-1.7423\end{array}$ & $\begin{array}{l}1.5401 \\
-1.5401\end{array}$ & $\begin{array}{c}1.7423 \\
-1.3426\end{array}$ & $\begin{array}{c}1.9492 \\
-1.1496\end{array}$ & $\begin{array}{l}1.3198 \\
-1.4728\end{array}$ & $\begin{array}{l}1.0279 \\
-1.1044\end{array}$ & $\begin{array}{l}0.9561 \\
-0.9561\end{array}$ & $\begin{array}{c}1.1044 \\
-1.0279\end{array}$ & $\begin{array}{c}1.4728 \\
-1.3198\end{array}$ \\
\hline 0.5 & $\begin{array}{l}1.1306 \\
-1.9301\end{array}$ & $\begin{array}{l}1.3235 \\
-1.7233\end{array}$ & $\begin{array}{l}1.5211 \\
-1.5211\end{array}$ & $\begin{array}{c}1.7233 \\
-1.3235\end{array}$ & $\begin{array}{c}1.9301 \\
-1.1306\end{array}$ & $\begin{array}{l}1.2284 \\
-1.3815\end{array}$ & $\begin{array}{l}0.9366 \\
-1.0131\end{array}$ & $\begin{array}{l}0.8648 \\
-0.8648\end{array}$ & $\begin{array}{c}1.0131 \\
-0.9366\end{array}$ & $\begin{array}{c}1.3815 \\
-1.2284\end{array}$ \\
\hline 0.7 & $\begin{array}{l}1.1116 \\
-1.9111\end{array}$ & $\begin{array}{l}1.3045 \\
-1.7043\end{array}$ & $\begin{array}{l}1.5021 \\
-1.5021\end{array}$ & $\begin{array}{c}1.7043 \\
-1.3045\end{array}$ & $\begin{array}{c}1.9111 \\
-1.1116\end{array}$ & $\begin{array}{l}1.1371 \\
-1.2901\end{array}$ & $\begin{array}{l}0.8452 \\
-0.9217\end{array}$ & $\begin{array}{c}0.7734 \\
-0.7734\end{array}$ & $\begin{array}{c}0.9217 \\
-0.8452\end{array}$ & $\begin{array}{c}1.2901 \\
-1.1371\end{array}$ \\
\hline 0.9 & $\begin{array}{l}1.0926 \\
-1.8921 \\
\end{array}$ & $\begin{array}{l}1.2855 \\
-1.6853 \\
\end{array}$ & $\begin{array}{l}1.4831 \\
-1.4831 \\
\end{array}$ & $\begin{array}{r}1.6853 \\
-1.2855 \\
\end{array}$ & $\begin{array}{c}1.8921 \\
-1.0926 \\
\end{array}$ & $\begin{array}{l}1.0458 \\
-1.1988 \\
\end{array}$ & $\begin{array}{l}0.7539 \\
-0.8304 \\
\end{array}$ & $\begin{array}{l}0.6821 \\
-0.6821 \\
\end{array}$ & $\begin{array}{r}0.8304 \\
-0.7539 \\
\end{array}$ & $\begin{array}{r}1.1988 \\
-1.0458 \\
\end{array}$ \\
\hline
\end{tabular}

Table-4: Upper and lower Control Limits for Non-Normal Population with Known cv Under Markoff's model $\left(\alpha_{1}=0.8\right)$

\begin{tabular}{|c|c|c|c|c|c|c|c|c|c|c|}
\hline \multicolumn{6}{|c|}{$(\mathrm{v}=\mathbf{0})$} & \multicolumn{5}{|c|}{$(\mathrm{v}=2)$} \\
\hline $\begin{array}{r}\lambda_{3} \rightarrow \\
\lambda_{4} \downarrow\end{array}$ & -1 & -0.5 & $\mathbf{0}$ & 0.5 & 1 & -1 & -0.5 & $\mathbf{0}$ & 0.5 & 1 \\
\hline \multirow{2}{*}{-0.5} & 0.6715 & 3.1847 & 4.7768 & 5.4479 & 5.1980 & 0.7952 & 1.9013 & 2.6341 & 2.9936 & 2.9798 \\
\hline & 1980 & -5.4479 & -4.7768 & -3.18 & -0.6 & -2.9798 & -2.9 & & -1.9013 & -0.7952 \\
\hline \multirow{2}{*}{-0.3} & 0.9428 & 3.4559 & 5.0480 & 5.7191 & 5.4692 & 0.8977 & 2.0037 & 2.7365 & 3.0960 & 3.0822 \\
\hline & -5.4692 & -5.7191 & -5.0480 & -3.4559 & -0.9428 & -3.0822 & -3.0960 & -2.7365 & -2.0037 & -0.8977 \\
\hline \multirow{2}{*}{-0.1} & 1.2140 & 3.7271 & 5.3192 & 5.9904 & 5.7405 & 1.0001 & 2.1061 & 2.8389 & 3.1984 & 3.1846 \\
\hline & -5.7405 & -5.9904 & -5.3192 & -3.7271 & -1.2140 & -3.1846 & -3.1984 & -2.8389 & -2.1061 & -1.0001 \\
\hline \multirow{2}{*}{0} & 1.3496 & 3.8627 & 5.454 & 6.1260 & 5.8761 & 1.0513 & 2.1573 & 2.8901 & 3.2496 & 3.2358 \\
\hline & -5.8761 & -6.1260 & -5.4 & -3.8627 & -1.3496 & -3.2358 & -3.2496 & -2.8901 & -2.1573 & -1.0513 \\
\hline \multirow{2}{*}{0.1} & 1.4852 & 3.9983 & 5.5905 & 6.2616 & 6.0117 & 1.1025 & 2.2085 & 2.9413 & 3.3008 & 3.2870 \\
\hline & -6.0117 & -6.2616 & -5.5905 & -3.9983 & -1.4852 & -3.2870 & -3.3008 & -2.9413 & -2.2085 & -1.1025 \\
\hline \multirow{2}{*}{0.3} & 1.7565 & 4.2696 & 5.8617 & 6.5328 & 6.2829 & 1.2049 & 2.3110 & 3.0437 & 3.4032 & 3.3895 \\
\hline & -6.2829 & -6.5328 & -5.8617 & -4.2696 & -1.7565 & -3.3895 & -3.4032 & -3.0437 & -2.3110 & -1.2049 \\
\hline \multirow{2}{*}{0.5} & 2.0277 & 4.5408 & 6.1329 & 6.8040 & 6.5542 & 1.3073 & 2.4134 & 3.1462 & 3.5057 & 3.4919 \\
\hline & -6.5542 & -6.8040 & -6.1329 & -4.5408 & -2.0277 & -3.4919 & -3.5057 & -3.1462 & -2.4134 & -1.3073 \\
\hline \multirow{2}{*}{0.7} & 2.2989 & 4.8120 & 6.4042 & 7.0753 & 6.8254 & 1.4097 & 2.5158 & 3.2486 & 3.6081 & 3.5943 \\
\hline & -6.8254 & -7.0753 & -6.4042 & -4.8120 & -2.2989 & -3.5943 & -3.6081 & -3.2486 & -2.5158 & -1.4097 \\
\hline \multirow{2}{*}{0.9} & 2.5701 & 5.0833 & 6.6754 & 7.3465 & 7.0966 & 1.5122 & 2.6182 & 3.3510 & 3.7105 & 3.6967 \\
\hline & -7.0966 & -7.3465 & -6.6754 & -5.0833 & -2.5701 & -3.6967 & -3.7105 & -3.3510 & -2.6182 & -1.5122 \\
\hline
\end{tabular}

\begin{tabular}{|c|c|c|c|c|c|c|c|c|c|c|}
\hline \multicolumn{9}{|c|}{$(\mathbf{v}=\mathbf{4})$} & \multicolumn{5}{c|}{ (v=6) } \\
\hline$\lambda_{\mathbf{3}} \rightarrow$ & $\mathbf{- 1}$ & $\mathbf{- 0 . 5}$ & $\mathbf{0}$ & $\mathbf{0 . 5}$ & $\mathbf{1}$ & $\mathbf{- 1}$ & $\mathbf{- 0 . 5}$ & $\mathbf{0}$ & $\mathbf{0 . 5}$ & $\mathbf{1}$ \\
$\boldsymbol{\lambda}_{\mathbf{4} \downarrow}$ & & & & & & & & & & \\
\hline $\mathbf{- 0 . 5}$ & 1.1894 & 1.4312 & 1.6565 & 1.8655 & 2.0579 & 1.6587 & 1.3903 & 1.3313 & 1.4816 & 1.8414 \\
& -2.0579 & -1.8655 & -1.6565 & -1.4312 & -1.1894 & -1.8414 & -1.4816 & -1.3313 & -1.3903 & -1.6587 \\
\hline
\end{tabular}




\begin{tabular}{|c|c|c|c|c|c|c|c|c|c|c|}
\hline-0.3 & $\begin{array}{r}1.1773 \\
-2.0459 \\
\end{array}$ & $\begin{array}{r}1.4191 \\
-1.8534 \\
\end{array}$ & $\begin{array}{l}1.6445 \\
-1.6445 \\
\end{array}$ & $\begin{array}{r}1.8534 \\
-1.4191 \\
\end{array}$ & $\begin{array}{r}2.0459 \\
-1.1773 \\
\end{array}$ & $\begin{array}{r}1.5710 \\
-1.7538 \\
\end{array}$ & $\begin{array}{r}1.3026 \\
-1.3940 \\
\end{array}$ & $\begin{array}{l}1.2436 \\
-1.2436 \\
\end{array}$ & $\begin{array}{r}1.3940 \\
-1.3026 \\
\end{array}$ & $\begin{array}{r}1.7538 \\
-1.5710 \\
\end{array}$ \\
\hline \multirow[b]{2}{*}{-0.1} & 1.1652 & 1.4070 & 1.6324 & 1.8413 & 2.0338 & 1.4833 & 1.2149 & 1.1559 & 1.3063 & 1.6661 \\
\hline & -2.0338 & -1.8413 & -1.6324 & -1.4070 & -1.1652 & -1.6661 & -1.3063 & -1.1559 & -1.2149 & -1.4833 \\
\hline \multirow{2}{*}{$\mathbf{0}$} & 1.1592 & 1.4010 & 1.6264 & 1.8353 & 2.0278 & 1.4395 & 1.1711 & 1.1121 & 1.2624 & 1.6222 \\
\hline & -2.0278 & -1.8353 & -1.6264 & -1.4010 & 92 & -1.6222 & -1.2624 & -1.1121 & -1.1711 & -1.4395 \\
\hline \multirow{2}{*}{0.1} & 1.1532 & 1.3950 & 1.6203 & 1.8293 & 2.0217 & 1.3956 & 1.1272 & 1.0682 & 1.2186 & 1.5784 \\
\hline & -2.0217 & -1.8293 & -1.6203 & -1.3950 & -1.1532 & -1.5784 & -1.2186 & -1.0682 & -1.1272 & -1.3956 \\
\hline \multirow{2}{*}{0.3} & 1.1411 & 1.3829 & 1.6083 & 1.8172 & 2.0097 & 1.3080 & 1.0395 & 0.9805 & 1.1309 & 1.4907 \\
\hline & -2.0097 & -1.8172 & -1.6083 & -1.3829 & -1.1411 & -1.4907 & -1.1309 & -0.9805 & -1.0395 & -1.3080 \\
\hline \multirow{2}{*}{0.5} & 1.1290 & 1.3708 & 1.5962 & 1.8051 & 1.9976 & 1.2203 & 0.9519 & 0.8928 & 1.0432 & 1.4030 \\
\hline & -1.9976 & -1.8051 & -1.5962 & -1.3708 & -1.1290 & -1.4030 & -1.0432 & -0.8928 & 519 & -1.2203 \\
\hline \multirow{2}{*}{0.7} & 1.1170 & 1.3588 & 1.5841 & 1.7930 & 1.9855 & 1.1326 & 0.8642 & 0.8052 & 0.9555 & 1.3153 \\
\hline & -1.9855 & -1.7930 & -1.5841 & -1.3588 & -1.1170 & -1.3153 & -0.9555 & -0.8052 & -0.8642 & -1.1326 \\
\hline \multirow{2}{*}{0.9} & 1.1049 & 1.3467 & 1.5721 & 1.7810 & 1.9735 & 1.0458 & 0.7539 & 0.6821 & 0.8304 & 1.1988 \\
\hline & -1.9735 & -1.7810 & -1.5721 & -1.3467 & -1.1049 & -1.1988 & -0.8304 & -0.6821 & -0.7539 & -1.0458 \\
\hline
\end{tabular}

\section{CONCLUSION}

Thus we see that either the values of the upper limits or lower limits will serve the purpose. Just to avoid negative sign it is advisable to tabulate values of upper limits, since lower limits have always negative values. However, when the autocorrelation is high in the process, the control limits feature is worthless in the detection ability of the chart, and the advantage of the mean chart is reduced. Non-normality parameters are less effective than the Markoff's process.

\section{REFERENCES}

[1]. Abraham, B. and Kartha, C.P. (1979). "Forecast stability and control Charts", ASQC Technical Conference Transactions. American Society for Quality Control, Milwaukee, WI, pp 675-685.

[2]. Alwan, L.C. (1991). “Autocorrelations: fixed and versus variable controlLimits", Qual. Eng 4:167-188.

[3]. Alwan, L.C. and Roberts, H.V. (1988). "Time-series modeling for statistical process control”, J Bus Econ Stat 6:87-95.

[4]. Barton, D. E. and Dennis, K. E. (1952). "The condition under whichGram-Charlier and Edgeworth curve are positive definite and unimodel", Biometrika, 39, 425-427.

[5]. Bennett, C. (1954) "Effect of measurement error on chemical process Control", Industrial Quality Control 10:17-20.

[6]. Berthouex, P.M., Hunter,W.G. and Pallesen, L. (1978). "Monitoring sewage treatment plants: some quality control aspects", J Qual. Technol. 10:139-149.

[7]. Box, G., Jenkins, G. and Reinsel, G. (1994). “Time Series Analysis, Forecasting and Control”, Prentis-Hall, Englewood Cliffs, New Jersey

[8]. Bhayare, U. and Singh, J. R. (2017). "The Effect of Measurement Error on the Power of X Charts with Known Coefficient of variation", International Journal of Scientific Research in Mathematical and Statistical Sciences, Vol.4, Issue.5, pp.33-39.

[9]. Delves, L.M. and Mohamed, J.L. (1985). "Computational methods for integral equations", Cambridge University Press, New York, NY.

[10]. Dooley, K.J., Kapoor, S.G., Dessouky, M.I. and Devor, R.E. (1986). "An integrated quality systems approach to quality and productivity improvement in continuous manufacturing processes", Trans ASME J Eng Ind 108:322-327.

[11]. Ermer, D.S. (1980). "A control chart for dependent data", ASQC Technical Conference Transactions. American Society for Quality Control, Milwaukee, WI, pp 121-128.

[12]. Harris, Y.J. and Ross, W.H. (1991). "Statistical process control procedures for correlated observations”, Can J Chem Eng 69:48-57.

[13]. Kanazuka, T. (1986). "The effect of measurement error on the power of $X$ - $R$ charts", Journal of Quality Technology 18:91-95.

[14]. Kramer, H. and Schmid, W. (1997). "Control charts for time series", Nonlinear Anal 30:4007-4016.

[15]. Lin, W.S. and Adams, B.M. (1996). “Combined control charts for forecast based monitoring schemes”, J Qual. Technol. 28:289-301 .

[16]. Longnecker, M.T. and Ryan, T.P. (1992). "Charting correlated process data", Technical report no. 166. Department of Statistics, Texas A\&M University, College Station, Texas.

[17]. Lu, C. and Reynolds, M. (1999). "EWMA control charts for monitoring the mean of autocorrelated processes". J Qual. Technol. 31:166-188

[18]. Mittag, H. (1995). "Measurement error effect on control chart performance", ASQC Annual Proceedings 49:66-73.

[19]. Mittag, H. and Stemmann, D. (1998). "Gauge imprecision effect on the performance of the $X$-S chart", Journal of Applied Statistics 25:307-

[20]. Mizuno, S. (1961). "Problems of measurement errors in process control", Bulletin of the International Statistical Institute 38:405-415

[21]. Montgomery, D.C. (1996). "Introduction to statistical quality control”, 3 rd edn. Wiley, New York.

[22]. Montgomery, D.C. and Mastrangelo, C.M. (1991). "Some statistical process control methods for autocorrelated data", J Qual. Technol. 23:179193

[23]. Padgett, C.S., Thombs, L.A. and Padgett, W.J. (1992). “On the $\alpha$-risks for Shewhart control charts", Commun Stat Simul Comput 21:11251147. 
[24]. Rahim, A.(1985). "Economic model of $X$ charts under non-normality and measurement errors", Computer and Operations Research 12:291-299.

[25]. Reynolds, M.R. JR., Arnold, J.C. and Baik, J.W. (1996). "Variable sampling interval in the presence of correlation", Journal of Quality Technology 28:12-30.

[26]. Runger, G.C., Willemain, T.R. and Prabhu, S. (1995). “Average run lengths for CUSUM control charts applied to residuals”, Commun Stat Theory Methods 24:273-282.

[27]. Schmid, W. (1995). "On the run length of a Shewhart chart for correlated Data", Stat Papers 36:111-130.

[28]. Schmid, W. (1997). "CUSUM control schemes for Gaussian processes”, Stat Papers 38:191-217.

[29]. Schmid, W. (1997b). "On EWMA charts for time series. In: Lenz HJ, Wilrich P-Th (eds) Frontiers of statistical quality control”, vol. 5, PhysicaVerlag, Heidelberg.

[30]. Schmid, W. and Schone, A. (1997). "Some properties of the EWMA control chart in the presence of autocorrelation", Ann Stat 25:1277-1283.

[31]. Searls, D. T. (1964). "The utilization of a known coefficient of variation in the estimation procedure", Journal of the American Statistical Association, 59, 1225-1226.

[32]. Shewhart, W.A. (1931). "Economic control of quality of manufactured product". Van Nostrand, New York..

[33]. Timmer, D.H., Pigantkiello, J. Jr. and Longnecker, M. (1998). "The development and evaluation of CUSUM-based control charts for an AR(1) process", IIE Trans 30:525-534.

[34]. Vander Weil, S.A. (1996). "Modeling processes that wander using movingaverage models", Technometrics 38:139-151.

[35]. Wade, R. and Woodall, W. (1993). “A review and analysis of cause-selecting control charts", J Qual. Technol. 25:161-169.

[36]. Wardell, D.G., Moskowitz, H. and Plante, R.D. RD (1992). "Control charts in the presence of data correlation", Manage Sci 38:1084-1105.

[37]. Wardell, D.G., Moskowitz, H. and Plante, R.D. (1994). "Run length distributions of special-cause control charts for correlated processes", Technometrics 36:3-17.

[38]. Yang, S. (2002). "The effects of imprecise measurement on the economicasymmetric $X$ and S control charts", The Asian Journal on Quality 3:46-56.

[39]. Yashchin, E. (1993). "Performance of CUSUM control schemes for serially correlated observations", Technometrics 35:37-52

[40]. Zhang, N.F. (1997). "Detection capability of residual control chart for stationary process data", J Appl. Stat. 24:363-380.

[41].Zhang, G. (1984). "A new type of control charts and a theory of diagnosis with control charts", World Quality Congress Transactions, AmericanSociety for Quality Control, Milwaukee, WI, pp 75-85. 